\title{
Original
}

\section{Recidiva local del tumor renal}

\author{
Emilio García Galisteo, P. Morales Jiménez, D. Hernández Alcaraz, Víctor Baena González \\ Servicio de Urología. Hospital Universitario Carlos Haya. Málaga. España.
}

$\mathrm{E}_{\mathrm{e}}^{1}$ carcinoma de células renales (CCR) constituye 3\% de todas las neoplasias malignas del adulto, representando el tercer tumor, en cuanto a prevalencia, de los tumores urológicos, siendo el más letal de los mismos. En España la incidencia está en torno a 4,5 por 100.000 habitantes y en Estados Unidos se estima que este año serán diagnosticados más de 54.000 personas y habrá en torno a 13.000 muertes como consecuencia de este tumor. Su incidencia ha ido aumentando aproximadamente un $2 \%$ anual probablemente en relación con el avance en las técnicas de imagen que permiten en muchas ocasiones detectarlo de forma incidental cuando se realiza un estudio por otro motivo. El CCR es más frecuente en medio urbano que rural con una relación entre sexos (varón/ mujer) de 3:2, su incidencia aumenta con la edad, siendo más frecuente entre las quinta y séptima década de la vida $^{1}$.

Después del tratamiento quirúrgico, en pacientes con tumores localizados, se producen un 20$30 \%$ de recidivas o metástasis, siendo el pulmón el órgano más frecuentemente afectado (50-60\% de los casos) $^{2}$. La presencia de metástasis después de la cirugía ocurre generalmente en los 3 primeros años tras la cirugía. El intervalo libre de enfermedad entre el diagnóstico y la detección de la enfermedad metastásica están muy relacionados con la supervivencia. De tal forma que los pacientes que tienen mayor intervalo libre de enfermedad tienen una supervivencia mayor.

\section{Definición y prevalencia de la recidiva local}

La recidiva local de un CCR tras una nefrectomía radical consiste en la aparición de una metástasis del CCR en la fosa renal. Aunque la presencia de recurrencia local en sujetos con enfermedad renal metastásica puede encontrarse hasta en un $66 \%{ }^{3}$, la recidiva del CCR en la fosa renal en ausencia de metástasis en otra localización es una eventualidad rara, su prevalencia va del 0,7 al $1,8 \% \%^{4,5}$.

La recidiva del CCR tras una cirugía ahorradora de nefronas se define como aparición de nuevos tumores renales en una zona distante al tumor renal primario.

Entre las teorias que intentan explicar por qué ocurre esta recidiva están: la aparición de una metástasis en la glándula suprarrenal ipsilateral que se dejó durante la nefrectomía, la persistencia de metástasis tumorales en ganglios linfáticos generalmente extrahiliares, la persistencia de restos tumorales en restos de la fascia de Gerota o del músculo psoas y la implantación de células tumorales en el lecho quirúrgico por una inadecuada técnica quirúrgica ${ }^{6}$.

La recidiva local en pacientes sometidos a una cirugía ahorradora de nefronas puede ocurrir por persistencia de restos tumorales que no han sido extirpados correctamente y se ha dejado un margen quirúrgico positivo $\mathrm{y}$ por la presencia de multifocalidad tumoral. La multifocalidad tumoral hay que valorarla con cuidado porque se ha visto que la presencia de pequeños focos microscópicos de células tumorales en otras partes del riñón no necesariamente van a condicionar el desarrollo de recidivas locales. En la Tabla 1 vemos la prevalencia de multifocalidad de diferentes series incluyendo pacientes con tumores pequeños menores de $4 \mathrm{~cm}^{7}$.

Aunque siempre que se realice una cirugía preservadora de nefronas se debe tener en cuenta el riesgo de recidiva, la relación de multifocalidad y recidiva local no es ni lineal ni previsible, como lo sugiere la baja tasa de recurrencia local después de cirugía. En más de 1.800 casos de cirugía conservadora reflejados en la literatura el riesgo de recurrencia local oscila entre el 0\% y 10\% y es claramente más baja en los pacientes que se sometieron a cirugía electiva con lesiones menores o iguales a $4 \mathrm{~cm}^{2}$ 
Tabla 1. Incidencia de carcinoma renal multifocal ${ }^{7}$

\begin{tabular}{|c|c|c|c|c|c|}
\hline Referencias & $N^{\circ}$ Pacientes & $\mathrm{N}^{\circ}$ tumores (\%) & Fuente & Sección (mm) & $\begin{array}{c}\mathrm{N}^{\circ} \text { tumores } \\
\text { multifocales } \leq 4 \mathrm{~cm}(\%)\end{array}$ \\
\hline Miller et al. & 245 & $69(28)$ & Nefrectomía & - & $6(8,7 \%)$ \\
\hline Wunderlich et al. & 104 & $13(12,5)$ & Autopsia & 5 & $13(100)$ \\
\hline Kinouchi et al. & 124 & $8(6,5)$ & Nefrectomia & 3 & $4(50)$ \\
\hline Whang et al. & 44 & $11(25)$ & Nefrectomía & 3 & - \\
\hline Oya et al. & 108 & $7(6,5)$ & Nefrectomía & 3 & $3(42,8)$ \\
\hline Gohji et al. & 64 & $10(15,6)$ & Nefrectomía & 3 & $7(70)$ \\
\hline Bilen et al. & 40 & $4(10)$ & Nefrectomía & 3 & 0 \\
\hline Herranz Amo et al. & 110 & $13(11,8)$ & Nefrectomía & - & - \\
\hline Kletscher et al. & 100 & $16(16)$ & Nefrectomía & 3 & $8(50)$ \\
\hline Cheng et al. & 100 & $7(7)$ & Nefrectomía & 3 & $2(28,6)$ \\
\hline Mukamel at al. & 66 & $13(19,7)$ & Nefrectomía & - & $4(30,8)$ \\
\hline Nissenkorrn and Bernheim & 27 & $3(11,1)$ & Nefrectomía & 3 & $1(33)$ \\
\hline Barbalias et al. & 48 & $5(10,4)$ & Nefrectomía & - & - \\
\hline Total & 1180 & $179(15,2)$ & & & $48(4,9)$ \\
\hline
\end{tabular}

(Tabla 2) ${ }^{7}$. Fergany ${ }^{8}$ en el seguimiento de 117 pacientes tratados con cirugía ahorradora de nefronas, la tasa de recurrencia local fue del 10 y $12 \%$ para los T3a y T3b con un seguimiento de 105 y 79,5 meses respectivamente.

\section{Grupos de riesgo}

Entre los factores pronósticos que parecen aumentar el riesgo de recidiva local podemos destacar:

\section{Anatómicos}

Parece ser que los pacientes que más riesgo tienen de tener una recurrencia local de un CCR son los pacientes con mayor estadio patológico, ganglios positivos y cuando el tumor se extiende más allá de la fascia de Gerota y por tanto hay más posibilidades de tener márgenes positivos o restos celulares diseminados que puedan implantar en la fosa renal $^{9}$. Sin embargo existen series donde la recurrencia local está presente en estadíos niciales (T1T2) $)^{5,10,11}$

\section{Histológicos}

El factor más importante es el grado histológico de Fuhrman, subtipos histológicos recogidos en la clasificación de la OMS de 2004, la presencia de diferenciación sarcomatoide, invasión microvascular, necrosis tumoral y la afectación del sistema colector.

\section{Clínicos}

El estado funcional del paciente (teniendo en cuenta factores como la obesidad, tabaquismo, etc....) la presencia de síntomas localizados, caquexia, anemia, alteración plaquetaria.

\section{Moleculares}

Existen numerosos marcadores moleculares actualmente en investigación, incluyendo: la anhidrasa carbónica IX (CAIX), factor vascular de crecimiento endotelial (VEGF), factor inducible de hipoxia (HIF), el Ki67, p53, PTEN, E-cadherin, y CD44. Hasta ahora, no se recomienda su uso generalizado. Recientemente, los perfiles de expresión génica han identificado 259 genes que predicen la supervivencia de forma independiente de la clínica, en tumores de células renales convencionales, siendo una línea de investigación importante.

El CCR heredo-familiar se asocian a la presencia de tumores renales bilaterales y múltiples, destacan, la enfermedad de Von Hippel-Lindau (VHL), cáncer papilar hereditario, esclerosis tuberosa, leiomiomatosis hereditaria, sindrome de Birt-HoggDubé, Cáncer familiar no VHL sin translocación del cromosoma 3 , cáncer familiar con translocación constitucional del cromosoma $3^{12}$.

\section{Intervalo libre de enfermedad}

Parece ser que los pacientes que han tenido un mayor intervalo de tiempo entre la nefrectomía y la 
Tabla 2: Seguimiento en pacientes sometidos a cirugía preservadora de nefronas por carcinoma renal esporádico ${ }^{7}$

\begin{tabular}{|c|c|c|c|c|}
\hline Referencias & $\mathbf{N}^{\circ}$ pacientes & $\begin{array}{c}\text { \% Supervivencia } \\
\text { enfermedad especifica }\end{array}$ & \% Recurrencia local & $\begin{array}{c}\text { Seguimiento medio } \\
\text { (meses) }\end{array}$ \\
\hline Jacobs et al. & 51 & 84 & 10 & 60 \\
\hline Marberger et al. & 72 & 78 & 8 & - \\
\hline Smith at al. & 39 & 72 & 8 & - \\
\hline Marshall y Walsh & 10 & 90 & 10 & 24,5 \\
\hline Bazeed at al. & 51 & 96 & 4 & 35,8 \\
\hline Carini et al. & 35 & 89 & 3 & 45,8 \\
\hline Gohji et al. & 21 & 100 & 0 & - \\
\hline Morgan y Zincke & 104 & 89 & 6 & 60 \\
\hline Selli et al. & 56 & 90 & 4 & 46,2 \\
\hline Provet at al. & 44 & 88 & 2 & 36 \\
\hline Steinbach at al. & 121 & 90 & 4,1 & 47 \\
\hline Moll et al. & 142 & 98 & 1,4 & 34,8 \\
\hline Thrasher et al. & 21 & 100 & 0 & 40 \\
\hline Lerner at al. & 185 & 89 & 5,9 & 44 \\
\hline D'Armiento at al. & 19 & 95 & 0 & 70 \\
\hline Indudhara et al. & 35 & 100 & 0 & 37 \\
\hline Van popel at al. & 76 & 96 & 0 & 75 \\
\hline Hafez et al. & 485 & 92 & 3,2 & 47 \\
\hline Barbalias et al. & 41 & 97,5 & 7,3 & 59 \\
\hline Belldegrun et al. & 146 & 93 & 2,7 & 74 \\
\hline Lee et al. & 79 & 96 & 0 & 40 \\
\hline Total (rango) & 1833 & $(72-100)$ & $(0-10)$ & $(24-75)$ \\
\hline
\end{tabular}

aparición de la recurrencia son los pacientes que tienen mejor pronóstico y mayor probabilidad de que tras el tratamiento de la recurrencia el paciente viva más ${ }^{5}$.

Resumiendo, los pacientes con mayor riesgo de tener recidiva local son los tumores avanzados y los que presentan ganglios positivos siendo la recurrencia local en pacientes con T1-2 NOMO rara.

\section{Nomogramas para predecir recidiva}

Se han desarrollado sistemas de pronóstico y nomogramas que combinan factores pronósticos independientes, para predecir la probabilidad de recurrencia tras el tratamiento del CCR (Fig. 1). Entre ellos podríamos destacar el nomogramas de Kattan ${ }^{13}$, el modelo de la UISS ${ }^{14}$ (Los Angeles Integrated Staging System), el modelo de Yaycigoglu ${ }^{15}$ y el de Cindolo ${ }^{16}$, todos ellos con resultados semejantes para sus poblaciones de estudio. Estos sistemas podrían ser

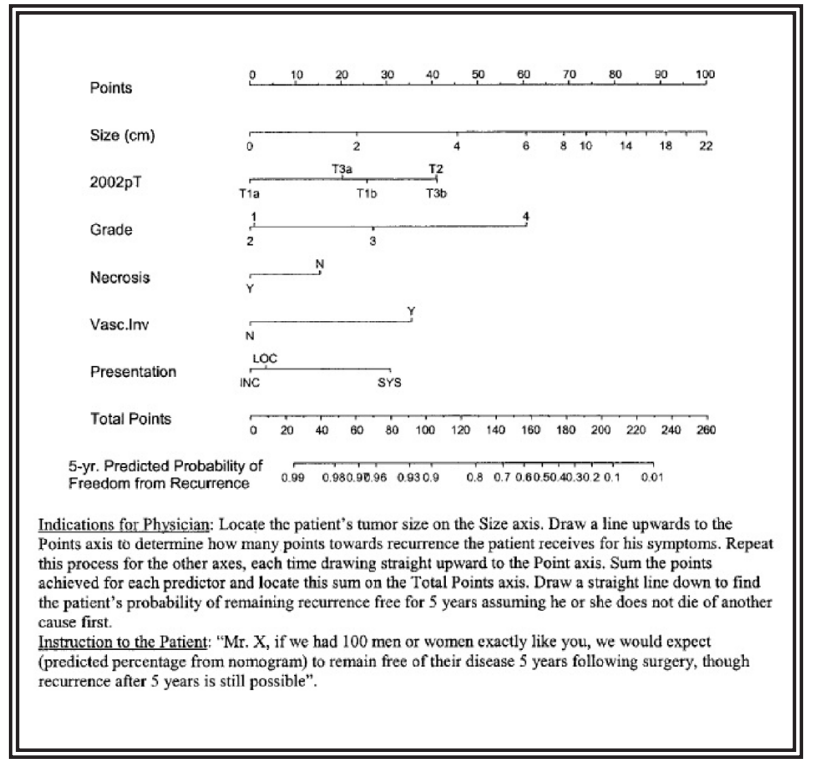

FIGURA 1. Nomograma para la recurrencia del carcinoma de células renales 
incluso más exactos que la clasificación TNM o el grado histológico de Fuhrman por sí solos para predecir la supervivencia. A pesar de ello, no se recomienda su uso, ya que hacen falta estudios prospectivos y aleatorizados más amplios para demostrar su superioridad. Proporcionan una predicción de pronóstico útil para los pacientes incluidos en ensayos clínicos. Tampoco se recomienda el uso de rutina de marcadores moleculares.

Para la práctica clínica se recomienda la utilización del sistema de clasificación TNM, el grado de Fuhrman y la clasificación de los subtipos de carcinoma de células renales. La Asociación Europea de Urología recomienda la utilización del sistema de clasificación de riesgo de la Clínica Mayo que estatifica el riesgo usando los factores $\mathrm{T}$ y $\mathrm{N}$ de la clasificación TNM, el tamaño tumoral, el grado nuclear de Fuhrman y la presencia o no de necrosis (Tabla 3 y 4) ${ }^{12,17-19}$.

\section{Seguimiento}

En el seguimiento de los pacientes es importante la detección precoz de la metástasis o la recidiva local y si el paciente está asintomático aún mejor. Se ha visto que las recurrencias en pacientes sintomáticos son de mayor tamaño, probablemente porque afectan a estructuras $u$ órganos

Tabla 3. Algoritmo para predecir la aparición de metástasis tras nefrectomía en pacientes con carcinoma de células claras según el Sistema de puntuación de la Clínica Mayo. (Bajo riesgo: 0-2; riesgo intermedio 3-5; alto riesgo>6)

\begin{tabular}{lc}
\hline & Puntuación \\
\hline Tumor primario & 0 \\
T1a & 2 \\
pT1b & 3 \\
pT2 & 4 \\
pT3-T4 & \\
Tamaño del tumor & 0 \\
$\quad<10 \mathrm{~cm}$ & 1 \\
$>10 \mathrm{~cm}$ & \\
& \\
Ganglios linfáticos regionales & 0 \\
pNx/pNO & 2 \\
pN1-pN2 & \\
Grado nuclear & \\
Grado 1-2 & 0 \\
Grado 3 & 1 \\
Grado 4 & 3 \\
Necrosis tumoral & \\
$\quad$ No necrosis & \\
Necrosis & 0 \\
\end{tabular}

Tabla 4. Riesgo acumulado de metástasis (\%) tras nefrectomía por CRR de acuerdo con el sistema de puntuación de la Clínica Mayo

\begin{tabular}{lcccc}
\hline Grupos de riesgo & $\mathbf{1}^{\circ}$ año & $\mathbf{2}^{\circ}$ año & $\mathbf{3}^{\circ}$ año & $\mathbf{4}^{\circ}$ año \\
\hline Riesgo bajo & 0,5 & 2,1 & 2,9 & 7,5 \\
Riesgo intermedio & 9,6 & 20,2 & 26,2 & 35,7 \\
Riesgo alto & 42,3 & 62,9 & 68,8 & 76,4 \\
\hline
\end{tabular}

adyacentes y en estos pacientes existe mayor probabilidad de que el tratamiento quirúrgico que se realice sea incompleto, existan márgenes positivos y en resumidas cuentas que el paciente presente una menor supervivencia ${ }^{9}$. Sin embargo en la literatura apenas existen directrices sobre el tipo de seguimiento que se debe realizar a pacientes que tienes mayor riesgo de recidiva local. La gran mayoría de las guías clínicas y publicaciones hacen referencia al seguimiento del carcinoma de celular renales (CCR) esporádico.

En cuanto al seguimiento en los pacientes con estadío clínico pT3 que son los que mayor probabilidad tienen de recurrencia local lo que más frecuentemente se recomienda es: valoración clínica, análisis sanguineo y $\mathrm{Rx}$ de tórax cada 6 meses durante 3 años y TC de abdomen cada 6 meses durante los 2-3 primeros años y posteriormente cada 2-3 años ${ }^{24}$.

Como todos sabemos los tumores renales hereditarios aparecen a edades más jóvenes y a menudo son multicéntricos y bilaterales, estos pacientes se enfrentan a un alto riesgo de recurrencia durante toda su vida. La mayoría de los CCR hereditarios parecen tener un mínimo potencial metastático cuando su tamaño es inferior a $3 \mathrm{~cm}$, como consecuencia se basa más en el tamaño que en la ubicación, histología o multifocalidad. En algunas publicaciones se recomienda que, los pacientes que presenten un fenotipo agresivo deberían someterse a pruebas de imagen cada 3-6 meses mientras los que presenten un fenotipo leve deberían realizarse cada 2-3 años ${ }^{20,24}$.

\section{Recomendaciones de las Guias Clínicas}

Al ser la recidiva local muy poco prevalente no existen recomendaciones generales para estos casos en la mayoría de las guías clínicas. La consideraciones que se establecen en estas guías hacen referencia a la indicación para realizar una cirugía conservadora de nefronas y a su seguimiento. 
a) La Asociación Europea de Urología, en su guía clínica, recomienda la realización de cirugía ahorradora de nefronas en tumores menores de $4 \mathrm{~cm}$ de diámetro mayor, y entre 4 y $7 \mathrm{~cm}$ en centros con experiencia en la realización de estos casos, siendo la vía de abordaje la laparoscópica para aquellos centros con amplia experiencia en esta técnica. No hace referencia al manejo de la recidiva después de la cirugía. En cuanto al seguimiento no hace referencia de forma especifica a la cirugía conservadora, se limita establecer revisiones en función de la estratificación del riesgo realizada mediante el sistema de clasificación y predicción del riego de metástasis de la Clínica Mayo ${ }^{12}$.

b) La Asociación Canadiense de Urología hace unas recomendaciones en cuanto a las indicaciones de la cirugía similares a la europea. Para los paciente sometidos a cirugía preservadora de neuronas establece el mismo tipo de seguimiento que para todos los pacientes clasificados como pT1 en la clasificación TNM, incluyendo en las revisiones una evaluación clínica bioquímica sanguínea y radiografía de Tórax cada año, la realización de un TC abdominal a los 24 y 60 meses. Es opcional la realización de un TC abdominal a los 3 meses de la cirugía y una ecografía abdominal anual para el grupo de nefrectomía parcial ${ }^{21}$.

c) The National Comprehensive Cancer Network (NCCN) establece las mismas indicaciones de tratamiento que las anteriores y recomienda el mismo seguimiento que los 3 primeros estadios semestralmente durante los 2 primeros años y posteriormente anualmente durante 5 años realizar evaluación clínica, analítica con bioquímica y LDH así como TC toracoabdominal $^{22}$.

\section{TRATAMIENTO}

El tratamiento para estos pacientes con recurrencia local tras tratamiento radical es la exéresis de la masa tumoral residual. Hay que tener en cuenta que estos paciente suelen tener infiltración de órganos vecinos por lo que la cirugía suele ser un proceso complejo y delicado. Sin embargo la exéresis de estas masas residuales puede aumentar la supervivencia en estos pacientes. Para los pacientes que no son candidatos a la cirugía la radioterapia puede ser una alternativa para el tratamiento de los sintomas.

El tratamiento de estos pacientes con recidiva local tras tratamiento parcial en ausencia de metástasis puede ser una nueva cirugía parcial, o si no es técnicamente posible, una nefrectomía radical generalmente con necesidad de diálisis inmediata y posteriormente alotrasplante.

En cuanto al tratamiento sistémico con quimioterapia o inmunoterapia decir que han ofrecido beneficio en la enfermedad metastásica renal pero no han aportado beneficio en cuanto a la recurrencia local $^{4}$. Además, la natural radiorresistencia de las células renales ha limitado mucho el papel de la radioterapia sistémica en el tratamiento de estos pacientes. Aunque el tratamiento sistémico no parece tener un papel importante en el tratamiento de la recidiva local del CCR, algunos autores creen que podría mejorar la supervivencia de los pacientes si se asocia al tratamiento quirúrgico ${ }^{9}$.

Sin embargo, el tratamiento sistémico exclusivamente no parece ser una buena alternativa; Fige G, en un estudio en 225 pacientes con CCR metastático tratados con interleuina-2 a altas dosis encontraron sólo un 14\% de respuestas, 5\% respuesta completa y un 9\% una respuesta parcial ${ }^{23}$. En los sujetos con recurrencia local de CCR no hubo ninguno con una respuesta completa y sólo 3 pacientes tuvieron una respuesta parcial.

El tratamiento quirúrgico con o sin tratamiento sistémico ha aumentado la supervivencia a 5 años a un 30-75\% 4,5,10,11 superando ampliamente la supervivencia que aporta el tratamiento médico (18\%) y la observación $(13 \%)^{4}$.

Por tanto parece que la única opción razonable hoy día que aporta beneficio en la supervivencia de estos pacientes es realizar un tratamiento quirúrgico de la metástasis local y de los órganos vecinos que pudieran estar afectados.

\section{REFERENCIAS}

1. Novick A, Campbell S. En: Campbell Urology. Tumores renales 8 ed. Buenos Aires: Medica Panamericana; 2004. p 2926.

2. Dekernion JB, Ramming KP, Smith RB. The natural history of metastatic renal cell carcinoma: a computer analysis. J Urol. 1978;120(2): 148-152.

3. Philips E, Messing EM. Role of lymphadenectomy in the treatment of renal cell carcinoma. Urology. 1993;41(1):9-15.

4. Itano NB, Blute ML, Spotts B, Zincke H. Outcome of isolated renal cell carcinoma fossa recurrence after nephrectomy. J Urol. 2000;164(2):322-325.

5. Schrodter S, Hakenberg OW, Manseck A, Leike S, Wirth MP. Outcome of surgical treatment of isolated local recurrence alter 928 radical nephrectomy for renal cell carcinoma. J Urol. 2002; 167(4):1630-1633.

6. Sandhu SS, Symes A, A'Hern R, Sohaib SA, Eisen T, Gore M, et al. Surgical excision of isolated renal-bed recurrence alter radical nephrectomy for renal cell carcinoma. BJU Int. 2005;95(4):522 525. 
7. Uzzo RG, Novick AC. Nephron Sparing Surgery for Renal Tumours: Indications, Tecniques and Outcomes. J Urol. 2001; 166(1):6-18.

8. Fergany AF, Hafez KS, Novick AC. Long-term results of nephron sparing surgery for localized renal cell carcinoma: 10-year followup. J Urol. 2000;163(2):442.

9. Levy DA, Slaton JW, Swanson DA, Dinney CP. Stage specific guidelines for surveillance after radical nephrectomy for local renal cell carcinoma. J Urol. 1998;159(4):1163-1167.

10. Tanguay S, Pisters LL, Lawrence DD, Dinney CP. Therapy of locally recurrent renal cell carcinoma after nephrectomy. J Urol. 1996;155(1):26-29.

11. Esrig D, Ahlering TE, Lieskovsky G, Skinner DG. Experience with fossa recurrence of renal cell carcinoma. J Urol. 1992;147 (6):1491-1494.
12. Ljungberg B, Hanbury DC, Kuczyk MA, Merseburger AS, Mulders $\mathrm{P}$, Patard JJ et al. Renal cell carcinoma guideline. European Urology 2007;51:1502-1510. (Traducción del artículo en Actas Urol Esp. 2009;33(3):270-279)

13. Sorbellini M, Kattan MW, Snyder ME, Reuter V, Motzer R, Goetzl M, et al. A postoperative prognostic nomograma predicting recurrence for patients with convencional clear cell renal cell carcinoma. J Urol. 2005;173(1):48-51.

Correspondencia autor: Dr. Emilio García Galisteo Servicio de Urología. Hospital Universitario Carlos Haya Avda. Carlos Haya, s/n - 29010 Málaga. Tel.: 951290000 E-mail autor: eggalisteo@yahoo.es Información artículo: Original Trabajo recibido: marzo 2009 Trabajo aceptado: abril 2009 